\title{
Could GRB 060614 and its presumed host galaxy be a chance superposition?
}

\author{
B. E. Cobb $^{1}$, C. D. Bailyn ${ }^{1}$, P. G. van Dokkum ${ }^{1}$, and P. Natarajan ${ }^{1}$ \\ cobb@astro. yale.edu
}

\begin{abstract}
The lack of an observed supernova associated with GRB 060614 appears to require a new paradigm for the formation of (a subset of) long-duration GRBs. This requirement is based on the presumed low redshift of the burst, which was inferred from the spatial coincidence of the afterglow with a $z=0.125$ galaxy. We explore the possibility that this low-redshift galaxy is a chance superposition along the line of sight to GRB 060614. We examine the galaxy distribution of the field of GRB 060614 and find that the probability of a chance association with a galaxy at least as bright as the putative host is only $\sim 0.5-1.9 \%$. However, for the current ensemble of $\approx 180$ Swift GRBs it is likely that several such coincidences have occurred, and given the "non-standard" nature of GRB 060614 it is not implausible that this is one such occurrence. Thus the conclusion that GRB 060614 requires a revision to the formation paradigm for long-duration GRBs should be approached with caution.
\end{abstract}

Subject headings: gamma rays: bursts

\section{Introduction}

The evidence that long-duration GRBs are associated with supernovae (SNe) is very strong. From observed low-luminosity GRBs with SNe that dominate the bursts' optical emission (e.g. GRB 980425/SN 1998bw and 060218/SN 2006aj; Galama et al. 1998; Cobb et al. 2006; Ferrero et al. 2006; Mirabal et al. 2006; Modjaz et al. 2006; Sollerman et al. 2006) to high-luminosity GRBs with SNe embedded in the bursts' optical afterglow (e.g. GRB 030329/SN 2003dh; Hjorth et al. 2003; Stanek et al. 2003; Bloom et al. 2004) has emerged a paradigm in which long-duration GRBs are produced by the core collapse supernovae of massive stars.

\footnotetext{
${ }^{1}$ Department of Astronomy, Yale University, P.O. Box 208101, New Haven, CT 06520
} 
The study of this association is difficult at high redshifts, due to the intrinsic faintness of the SNe when compared with the luminosities produced at all wavelengths by ultrarelativistic GRB jets. Any possible low-redshift GRB is, therefore, of great importance and is scrutinized by many observers for photometric or spectroscopic evidence of a SN. Such was the case for GRB 060614 (Parsons et al. 2006), a burst that was typical in most respects and was followed by a relatively bright and long-lasting optical afterglow (Holland 2006; Brown et al. 2006).

Initial observations of GRB 060614 detected afterglow light in all Swift UVOT bands, indicating that the GRB had a moderate redshift of $z<1.7$, due to the absence of absorption from the Lyman- $\alpha$ forest (Holland 2006; Schaefer \& Xiao 2006). From the spectral parameters of the GRB, Pelangeon \& Atteia (2006) calculated the pseudo-redshift of GRB 060614 to be $p z=1.45 \pm 0.85$, consistent with the earlier estimate (see also Schaefer \& Xiao 2006). Spectroscopic observations of the afterglow of GRB 060614 taken less than two days post-burst did not yield a spectroscopic redshift (Fugazza et al. 2006a), as no absorption or emission features were detected on top of the power-law spectrum of the afterglow.

More spectra were obtained at the afterglow position of GRB 060614 as the afterglow continued to fade away. In a spectrum taken $\sim 5$ days post-burst (Price et al. 2006), a single strong emission line was detected and interpreted to be due to $\mathrm{H} \alpha$ from the GRB host galaxy at $z=0.13$. The redshift of this potential host galaxy was confirmed to be $z=0.125$ by Fugazza et al. (2006b). With the possibility of this burst being at very low redshift, several observers continued careful follow-up campaigns (e.g. Brown \& Holland 2006; Cobb \& Bailyn 2006; Fynbo et al. 2006a), anticipating the brightening expected from a SN at that redshift. However, in time, all decaying afterglow light curves leveled off to a constant magnitude, interpreted to be the magnitude of the host galaxy, and no SN brightening was observed (Cobb \& Bailyn 2006; Della Valle et al. 2006; Fynbo et al. 2006b; Gal-Yam et al. 2006).

The lack of an observed SN from this seemingly low-redshift GRB can be explained in several ways:

1. A SN did occur but was not detected due to high line-of-sight extinction toward the GRB, most likely due to dust in the GRB's host galaxy. However there are no indications of the presence of a supernova in IR observations (Cobb \& Bailyn 2006) and also no indication of the presence of this reddening in the UV/optical/IR observations of the afterglow (Holland 2006; Della Valle et al. 2006; Fynbo et al. 2006b; Gal-Yam et al. 2006).

2. A SN did occur but was not detected because the SN was underluminous by more than 5 magnitudes. This would be unexpected, however, as all previous GRB-related SNe 
have luminosities that cluster within a magnitude of the peak brightness of SN 1998bw (Zeh et al. 2004; Cobb et al. 2006; Ferrero et al. 2006). While local non-GRB Type Ibc SNe do span a larger range of $\pm \sim 2$ magnitudes from the peak of SN 1998bw (Richardson et al. 2006), even the dimmest known Ibc SN should have been detectable in the absence of strong reddening.

3. No stellar core collapse occurred, or the core collapse did not result in a typical GRB "hypernova". These possibilities have now been suggested by several authors (Della Valle et al. 2006; Fynbo et al. 2006b; Gal-Yam et al. 2006) and require a paradigm shift in our understanding of the formation mechanism of GRBs or, at the very least, require the introduction of a new class of GRBs.

4. The proposed host galaxy of GRB 060614 is not the true host galaxy but, instead, represents the chance coincidence of a low-redshift galaxy intersecting the line of sight toward what is actually a moderate-redshift GRB (as suggested by Schaefer \& Xiao 2006). The SN, therefore, did occur but is undetectable given the redshift of the burst.

In some ways, the fourth possibility is the most appealing, since it requires no new formation channel for long-duration GRBs, and is consistent with some features of the GRB itself (Schaefer \& Xiao 2006). However, it requires a spatial coincidence between a foreground galaxy and the GRB. Here we analyze a deep optical image of the field of GRB 060614 to study the statistics of such a coincidence empirically. In agreement with Schaefer \& Xiao (2006), we find that a chance superposition is not implausible. While statistical arguments can never rigorously exclude or require such a coincidence, the drastic nature of the alternatives suggests that the possibility of a chance superposition should be seriously considered.

\section{Data}

Our data were obtained using the ANDICAM instrument mounted on the $1.3 \mathrm{~m}$ telescope at Cerro Tololo Inter-American Observatory ${ }^{1}$. This telescope is operated as part of the Small and Moderate Aperture Research Telescope System (SMARTS) consortium² ${ }^{2}$ Imaging was obtained approximately every other night between 2006 June 15 - July 25 UT, which is between 0.65 and 40.73 days post-burst. A number of observations were affected by clouds

\footnotetext{
${ }^{1}$ http://www.astronomy.ohio-state.edu/ANDICAM

${ }^{2}$ http://www.astro.yale.edu/smarts.
} 
and are not included in this analysis. In total, 13 useful nights worth of data were collected (see Table 1).

Each night's data set consisted of six individual 360 s I-band observations and $30 \times 60$ s J-band observations. The data were reduced in the same way as in Cobb et al. (2006) and combined to produce a single 36 minute I-band exposure and a 30 minute J-band exposure per night. I-band observations taken 0.65 and 1.66 days post-burst both contain bright afterglow light and the remaining 11 images show no indication of brightening due to a SN (e.g. Cobb \& Bailyn 2006) down to $22 \mathrm{mag}$ in I and $20 \mathrm{mag}$ in J (see Table 1 and Figure 1 ). This result is in agreement with the more stringent limits imposed by other works (Della Valle et al. 2006; Fynbo et al. 2006b; Gal-Yam et al. 2006).

The 11 I-band images taken from 2.66 to 40.73 days post-burst were combined to produce a single image of a 5!4 ×5!2 field approximately centered on GRB 060614. The I-band

photometry is calibrated to a number of secondary standard stars in the field of GRB 060614. The magnitude of these secondary standards was derived using Landolt standard star observations (Landolt 1992) taken on 7 of the photometric nights when GRB 060614 was observed. The image reaches a $3 \sigma$ limiting magnitude of $I \approx 23.3$. The proposed host galaxy has an observed magnitude of $I=22.08 \pm 0.09$.

All objects in the field were cataloged using SExtractor (Bertin \& Arnouts 1996). 348 objects were detected using a $2 \sigma$ detection threshold. Using SExtractor's neural network star/galaxy classifier, 85 objects with CLASS_STAR $>0.8$ were considered stars and not included in the following analysis. A section of our master image is shown in Figure 2 with SExtracted galaxies shown as green and magenta ellipses. The proposed host galaxy of GRB 060614 is highlighted in red.

\section{Probability of a Chance Superposition}

In our 28 square arcminute field of view, the position of the optical afterglow of GRB 060614 can be determined to within sub-pixel accuracy. We calculate the probability by dividing the number of pixels covered by galaxies by the total number of pixels. The total pixel area of our image that is covered by galaxies is found by summing the number of pixels contained in each individual SExtracted galaxy. The area of each galaxy can be computed in two ways. First, the galaxy area can be taken as the area of the SExtracted ellipse $(\pi \times 3 *$ A_IMAGE $\times 3 *$ B_IMAGE), where the multiplier 3 is chosen so that the ellipse is visually coincident with the extent of the galaxy (see Figure 2). Alternatively, the area can be given by the isophotal SExtractor parameter ISOAREAF_IMAGE. From the sum of 
the ellipse areas, the number of pixels contained in galaxies divided by the total number of pixels in the image produces a probability of $3.5 \%$ that the optical afterglow would land on a pixel contained in any galaxy in the field. The isophotal area measurement produces somewhat smaller area values for each galaxy, reducing the probability to $2.4 \%$.

Of course, some of the galaxies in this image are dimmer than the proposed host galaxy of GRB 060614, and we are concerned here specifically with the chance of a superposition with galaxies similar to or brighter than the proposed host. All galaxies that are detected at the $4.8 \sigma$ level are considered "bright" galaxies (these galaxies are indicated in Figure 2 by the green ellipses). The value of $4.8 \sigma$ is chosen because it is the highest threshold for which the proposed host of GRB 060614 is still SExtracted. Of the 263 galaxies initially detected, 95 of those objects are still detected at a significance of greater than $4.8 \sigma$. The isophotal area contained within only these bright galaxies represents $1.9 \%$ of the total pixels in the image. The number of galaxies in this sample is reduced to $36 \%$ of the original, but this corresponds to only a $21 \%$ drop in probability because each bright galaxy covers significantly more area than any given dim galaxy.

To more fully explore the possibility that GRB 060614 is a chance superposition with a foreground galaxy, it would be helpful to know the redshift of all the sources in the field. In the absence of this data, we can only assume for this field a typical galaxy redshift distribution. In a VVDS-CDFS galaxy sample limited to $I<22.1$ (Le Fèvre et al. 2004), $99 \%$ of galaxies have a redshift of $z \leq 1.5$, which is at the low end of the redshift range for GRB 060614 suggested by Schaefer \& Xiao (2006). This indicates that if the burst was randomly associated with any of these bright galaxies, then that bright galaxy would almost certainly be at a redshift lower than that proposed for the burst. However, it was only the lack of an observed SN that called attention to this burst. If the proposed host galaxy of GRB 060614 had been at a redshift beyond that for which a SN could reasonably have been detected, then the burst would not have been singled out as either curious or groundbreaking given the burst's otherwise unexceptional characteristics.

There exists spectroscopic and/or photometric evidence for a SN component in most other bursts with $z \lesssim 0.7$ (Zeh et al. 2004; Ferrero et al. 2006). However, at redshifts approaching $z \sim 0.7$, the degeneracy between distance and reddening would allow for the reasonable assumption that the $\mathrm{SN}$ was not observed due to moderate extinction by hostgalaxy dust. To some extent, reddening can be constrained by analysis of the X-ray to optical SED of the GRB afterglow. In the case of GRB 060614, this yields a low reddening value of only a few tenths of a magnitude (Della Valle et al. 2006). This assumes, however, that the GRB afterglow experiences the same reddening as the associated SN, which may not be the case given the collimated versus (near-)isotropic configuration of the GRB and SN 
emission, respectively. The exact redshift at which a SN non-detection would have elicited serious proposals of a new GRB mechanism is difficult to quantify, but we choose a value of $z=0.4$. At this redshift, the non-detection of a SN excess above the brightness of a $I \sim 22$ host galaxy requires that any $\mathrm{SN}$ be at least a magnitude fainter at peak than the typical GRB-SNe value of $I \sim-19$, assuming a low reddening value measured from SED analysis. Given that a variation of only a magnitude is not surprising for Type Ibc SNe in general, this limit is still reasonable because HST observations may be expected to place more stringent limits than ground-based observations by resolving the optical afterglow/SN location within the galaxy. In such a case, the SN would be observed as a deviation in the power-law decay of the optical afterglow. For example, a SN at $z=0.7$ could have been detected above the afterglow in the HST observations taken by Gal-Yam et al. (2006).

In our flux-limited sample of VVDS-CDFS galaxies, 65\% have a redshift of $z \leq 0.7$, and $25 \%$ have $z \leq 0.4$. If projected area is similar, or at least random, for all galaxies, then the area covered by those galaxies is $2 / 3$ or $1 / 4$ of the total covered area, respectively. The specific probability of a chance superposition with a galaxy that has a redshift low enough for a null-SN detection to be of interest is then $1.2 \%$ (for $z \leq 0.7$ ) or $0.5 \%$ (for $z \leq 0.4$ ). This

does not take into account, however, the true angular size of the individual galaxies. If the lowest redshift galaxies trend toward larger projected surface areas than higher- $z$ galaxies, then the drop in the chance superposition probability caused by this redshift cut will be somewhat reduced.

Thus, depending on one's assumptions of the redshift limit out to which the lack of an apparent SN would be flagged as important, a chance superposition for this particular GRB can be ruled out with $98.1-99.5 \%$ certainty.

\section{Discussion}

Although the probability for a chance superposition for any given GRB is small, we must also consider that a large number of GRB fields have now been observed in this manner. If we assume that the galaxy density in the field of GRB 060614 is not unusual (as appears to be the case - Cobb et al. in prep), then given the $\approx 180$ bursts observed by Swift, a chance overlap of a galaxy brighter than $I \sim 22$ and the precise position of a GRB would be expected for $\approx 1-4$ GRBs. The probability that no chance overlaps would be observed in the ensemble is quite small, at $3.2 \%$ to $41 \%$, assuming a probability of $1.9 \%$ to $0.5 \%$ for a chance superposition in any given image. Most such superpositions would not be noticed, however, because the typical foreground galaxy would be at a redshift comparable to that of the GRB. In contrast, GRB 060614 has generated great interest because the galaxy implies 
that the GRB is at an unusually low redshift.

The strongest host galaxy claims can only be made for GRBs with detected optical afterglows. Of the $\sim 80$ optical afterglows detected following Swift bursts, the detection of no chance overlaps would not be unexpected at a probability of $22 \%$ to $67 \%$. Note, however, that the position of GRBs without optical afterglows are determined from Swift XRT observations of X-ray afterglows, which have a spatial precision of $\sim 4$ ". Taking this larger positional uncertainty into account would significantly increase the probability of chance superpositions.

One additional feature of GRB 060614 is that it appears to be at the periphery of the galaxy (Gal-Yam et al. 2006). This is not expected for standard long-duration GRB formation mechanisms, since the star formation rate at the outskirts of galaxies is expected to be low. Indeed Gal-Yam et al. (2006) invoke the location of the GRB within the galaxy as a first clue to the nature of a new GRB formation paradigm. The chance superposition hypothesis is consistent with an off-center location of the GRB. With deeper observations it may be possible to confirm or refute the presence of a background galaxy, although we note that there is $\mathrm{a} \sim 1 / 4$ chance that the center of the true host galaxy will lie directly behind that of the $z=0.125$ galaxy (Bloom et al. 2002).

We note that any superposition with a foreground galaxy requires that the source be lensed. However, the low luminosity and hence inferred low mass for this particular galaxy suggests that the lensing induced magnification is less than $5 \%$, which is inconsequential. This lensing estimate is obtained using standard assumptions and modeling the galaxy as a singular isothermal sphere.

It has been suggested that GRB 060505 is also a low-redshift GRB with no SN (Fynbo et al. 2006b), although the observational data are more limited both in the gamma-rays (Schaefer \& Xiao 2006) and for the afterglow, for which no optical spectrum has been reported. Clearly the existence of a second "060614-like" object would significantly diminish the probability of chance superpositions. But this argument requires that the two objects be members of the same putative new category, and it is not clear that this could be the case. In particular, there are significant differences in the GRB characteristics of the two sources (GRB 060505 was much shorter-4 s versus 120 s-and weaker than GRB 060614; Palmer et al. 2006; Parsons et al. 2006) and GRB 060505 appears to be located in a star forming region of a galactic spiral arm, whereas GRB 060614 seems to be outside the main region of star formation. If, as seems likely, the inferred low redshifts of these two objects require different formation mechanisms from each other, then an explanation of either or both of them as a chance superposition remains plausible. 
We emphasize that an analysis of this kind is by nature not definitive. From this argument alone, it is not possible to claim that GRB 060614 is a chance superposition between a low- $z$ galaxy and a moderate- $z$ GRB; we only claim that there is a possibility of such an occurrence. The fact that galaxies as bright as the purported host of GRB 060614 cover several percent of the field, and that well over a hundred GRBs have now been observed by Swift, suggests that a chance superposition cannot be excluded. Indeed, over the ensemble of Swift bursts, the probability that such a coincidence would be observed in at least one case is high. The unusual combination of a low-redshift putative host and the lack of an associated supernova make GRB 060614 an intriguing candidate for a chance superposition. Thus the conclusion that GRB 060614 requires a "new paradigm" for GRB formation should be approached with caution.

We thank SMARTS observers D. Gonzalez and J. Espinoza for their dedication to observing this source and S. Tourtellotte for assistance with optical data reduction. This

work is supported by NSF Graduate Fellowship DGE0202738 to BEC and NSF/AST grant 0407063 and Swift grant NNG05GM63G to CDB.

\section{REFERENCES}

Bertin, E., \& Arnouts, S. 1996, A\&AS, 117, 393

Bloom, J. S., Kulkarni, S. R., \& Djorgovski, S. G. 2002, AJ, 123, 1111

Bloom, J. S., van Dokkum, P. G., Bailyn, C. D., Buxton, M. M., Kulkarni, S. R., \& Schmidt, B. P. 2004, AJ, 127, 252

Brown, P. J., Holland, S. T., Mangano, V., Parsons, A. M., \& Gehrels, N. 2006, GRB Coordinates Network, 5262

Brown, P. J., Holland, S. T. 2006, GRB Coordinates Network, 5286

Cobb, B. E., \& Bailyn, C. D. 2006, GRB Coordinates Network, 5282

Cobb, B. E., Bailyn, C. D., van Dokkum, P. G., \& Natarajan, P. 2006, ApJ, 645, L113

Della Valle, M. et al. 2006, (astro-ph/0608322)

Ferrero, P. et al. 2006, A\&A in press, (astro-ph/0605058)

Fugazza, D., et al. 2006, GRB Coordinates Network, 5271 
Fugazza, D., et al. 2006, GRB Coordinates Network, 5276

Fynbo, J. P. U., et al. 2006, GRB Coordinates Network, 5277

Fynbo, J. P. U. et al. 2006, (astro-ph/0608313)

Galama, T. J., et al. 1998, Nature, 395, 670

Gal-Yam, A. et al. 2006, (astro-ph/0608257)

Hjorth, J. et al. 2003, Nature, 423, 847

Holland, S. T. 2006, GRB Coordinates Network, 5255

Landolt, A. U. 1992, AJ, 104, 340

Le Fèvre, O., et al. 2004, A\&A, 428, 1043

Mirabal, N., Halpern, J. P., An, D., Thorstensen, J. R., \& Terndrup, D. M. 2006, ApJ, 643, L99

Modjaz, M., et al. 2006, ApJ, 645, L21

Parsons, A. M., et al. 2006, GRB Coordinates Network, 5252

Patat, F., et al. 2001, ApJ, 555, 900

Pelangeon, A., \& Atteia, J.-L. 2006, GRB Coordinates Network, 5265

Palmer, D., et al. 2006, GRB Coordinates Network, 5076

Price, P. A., Berger, E., \& Fox, D. B. 2006, GRB Coordinates Network, 5275

Richardson, D., Branch, D., \& Baron, E. 2006, AJ, 131, 2233

Schaefer, B. E. 2006, (astro-ph/0608441)

Skrutskie, M. F., et al. 2006, AJ, 131, 1163

Sollerman, J., et al. 2006, A\&A, 454, 503

Stanek, K. Z., et al. 2003, ApJ, 591, L17

Zeh, A., Klose, S., \& Hartmann, D. H. 2004, ApJ, 609, 952 
Table 1. SMARTS observations of GRB 060614 afterglow + purported host galaxy

\begin{tabular}{rrrc}
\hline \hline Observation UT date (2006) & Days after GRB $^{\mathrm{a}}$ & $\mathrm{I} \mathrm{mag}^{\mathrm{b}}$ & $\mathrm{J} \mathrm{mag}^{\mathrm{b}, c}$ \\
\hline June 15.1780 & 0.65 & $19.00 \pm 0.04$ & $18.39 \pm 0.11$ \\
June 16.1909 & 1.66 & $20.62 \pm 0.10$ & $19.43 \pm 0.19$ \\
June 17.1865 & 2.66 & $21.41 \pm 0.15$ & $>19.1$ \\
June 19.3164 & 4.79 & $>21.7$ & $>19.0$ \\
June 21.3072 & 6.78 & $>21.9$ & $>19.1$ \\
June 25.4049 & 10.87 & $>21.8$ & $>19.1$ \\
June 27.3962 & 12.87 & $22.06 \pm 0.14$ & $>19.8$ \\
June 29.3504 & 14.82 & $22.04 \pm 0.15$ & $>18.9$ \\
July 01.3760 & 16.85 & $>21.8$ & $>19.2$ \\
July 05.2827 & 20.75 & $>22.0$ & $>18.9$ \\
July 18.2019 & 33.67 & $22.13 \pm 0.13$ & $>19.9$ \\
July 20.1319 & 35.60 & $22.31 \pm 0.21$ & $>19.6$ \\
July 25.2559 & 40.73 & $22.09 \pm 0.14$ & $>20.0$ \\
\hline
\end{tabular}

a Mid-exposure time of images in days after the burst trigger, which occurred at 2006 June 14, 12:43:48 UT.

${ }^{\mathrm{b}}$ These values have not been corrected for Galactic extinction. $3 \sigma$ limiting magnitudes are reported for observations in which the galaxy is not significantly detected.

${ }^{\mathrm{c} J}$-band photometric calibration was performed using two 2MASS stars (Skrutskie et al. 2006) in the field. 
Photometry of the proposed host galaxy of GRB 060614

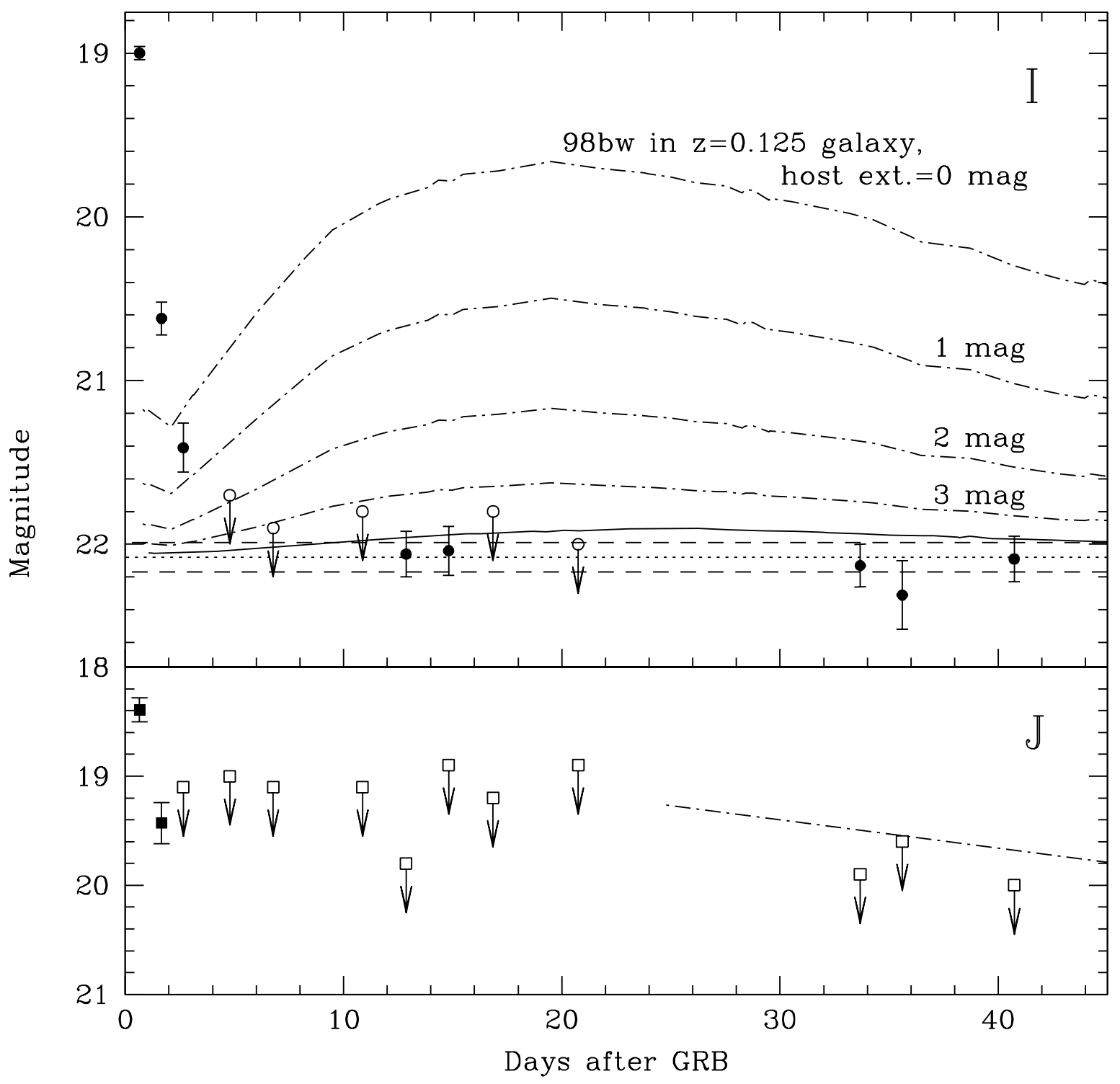

Fig. 1.- Photometry of the afterglow and proposed host galaxy of GRB 060614. Filled symbols represent detections; open symbols indicate $3 \sigma$ magnitude limits for observations in which the galaxy was not significantly detected. Top: I-band photometry, with the brightness of the galaxy determined from the master combined image $(I=22.08 \pm 0.09)$ indicated by the dotted line with $1 \sigma$ error (dashed lines). The dot-dashed lines indicate the expected brightness of the galaxy had a SN similar to SN 1998bw (Galama et al. 1998) occurred in the galaxy, assuming 0 to 3 magnitudes of host-galaxy extinction. Even with 3 magnitudes of extinction, at $z=0.125$ such a SN would have easily been observed. The solid line indicates a $z=0.5 \mathrm{SN} 1998 \mathrm{bw}$ superimposed on the galaxy; a SN with any higher redshift would be difficult to detect in our data. Note that other more stringent limits are available in the literature (Della Valle et al. 2006; Fynbo et al. 2006b; Gal-Yam et al. 2006). Bottom: J-band photometry; in the absence of the GRB afterglow, the galaxy remains undetected. A 1998bw-like SN would be expected to rouphly follow the dot-dashed line assuming the 


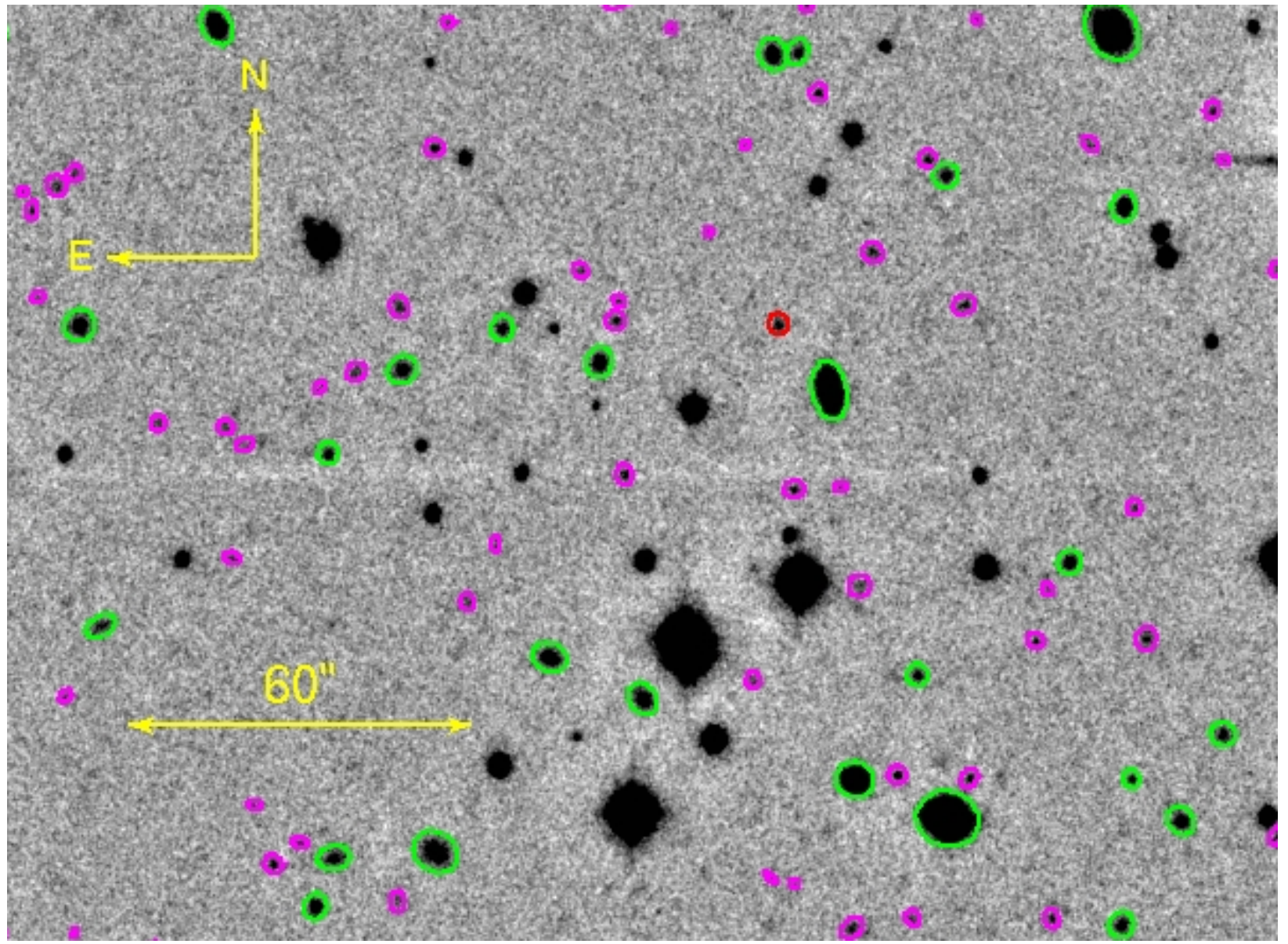

Fig. 2.- Section of the master image of GRB 060614. SExtracted galaxies are indicated in green and magenta. Each ellipse has axes $3^{*}$ A_IMAGE and $3^{*}$ B_IMAGE and position angle THETA_IMAGE. The proposed host galaxy of GRB 060614 is indicated in red. Bright galaxies are denoted by green ellipses; these are galaxies detected at the same or higher significance than the galaxy at the position of the optical afterglow of GRB 060614 . 